\title{
Characterization of Polyacrylamide Hydroxyapatite (PAHA) Disc Demineralization Invitro based on Optimized Areal Surface Profiling and Topography Analysis using Confocal Laser Scanning Microscopy
}

\author{
Eun-Jung Lee ${ }^{1,2 *}$ and Bo-Hyoung Jin ${ }^{2}$ \\ ${ }^{1}$ Department of Food \& Nutrition, College of Human Ecology, Seoul National University \\ ${ }^{2}$ Department of Preventive \& Social Dentistry, School of Dentistry, Seoul National University
}

Received: January 01, 2018; Published: February 09, 2018

*Corresponding author: Eun-Jung Lee, Department of Food \& Nutrition, College of Human Ecology, Seoul National University; Department of Preventive \& Social Dentistry, School of Dentistry, Seoul National University, South Korea, Fax-82-2-766-8781; Tel: 82-10-6425-0022; Email: leeej77@snu.ac.kr

Abstract

Objectives: The objective of this study was to characterize the degree of polyacrylamide hydroxyapatite (PAHA) disc demineralization in vitro based on both optimized areal surface profiling and topography analysis using confocal laser scanning microscopy (CLSM).

Methods: A total of 11 test samples were prepared using modified Association of Official Analytical Chemists (AOAC) methods. Test sample solution was incubated at $37^{\circ} \mathrm{C}$ for 150 minutes after inoculation with $1 \%$ Streptococcus mutans and radioisotope-labeled polyacrylamide hydroxyapatite (PAHA) discs. The degree of demineralization was measured based on ${ }^{32} \mathrm{P}$ released from each radioisotope-labeled PAHA disc using CLSM. Image analysis systems (2D and 3D) were used to determine surface topography and roughness.

Results: Sz (maximum height of the surface) showed a significant difference compared to conventional mean height parameters, such as Sa (mean height of the surface) and Sq (root square mean height). Regarding the skewness and kurtosis, distinct demineralization was observed for sponge cake $(\mathrm{p}<0.05)$. While these food samples showed minimal differences in released radioisotope ${ }^{32} \mathrm{P}$, a relatively distinct appearance was observed in CLSM.

Conclusions: Affinity with skewness (Ssk) and kurtosis (Sku) illustrated the predominance of peaks and valleys with the presence of inordinate peaks and valleys correspondingly. This study confirmed the feasibility of quantifying PAHA disc demineralization using both optimized areal surface profiling and surface topography analysis.

Keywords: Confocal laser scanning microscopy; Demineralization; Food; Polyacrylamide hydroxyapatite discs; Streptococcus mutans; Topography

Abbreviations: AFM: Atomic Force Microscopy; SEM: Scanning Electron Microscopy; KRIBB: Korea Research Institute Bioscience and Biotechnology; PAHA: Polyacrylamide Hydroxyapatite; CLSM: Confocal Laser Scanning Microscopy

\section{Introduction}

Because of its environmental aspects, serial buffering of the remineralization and demineralization process in tooth enamel is dependent on diet, reflecting the critical position of quantifying cariogenic potential of foods to prevent the development of dental caries. In 1970, the Food, Nutrition, and Dental Health program was started by American Dental Health Foundation to assess cariogenicity of foods. In 1999, methodology was updated, leading to the reliable assessment of the cariogenic potential of foods using animal models, plaque $\mathrm{pH}$ standards, and an in situ model for enamel demineralization [1]. This has laid down the basis to correlate dental caries development and food consumption [2-5]. New methods for estimating the cariogenic potential of foods has been developed, including profilometry, scanning electron microscopy (SEM), microradiography, digital image analysis, and atomic force microscopy (AFM) through surface characterization $[1,6,7]$.

The relative assessment of foods cariogenic potential offers a credible method of understanding the development of dental caries based on dietary ingredients and dental interaction. However, the results of absolute determination for the cariogenic potential 
of a wide range of foods remain inconsistent due to its complex ingredients in vivo [8]. Extrinsic factors have been widely studied using SEM, AFM, and CLSM in dental structure. There are no completely reliable techniques for quantifying the initial enamel demineralization in vitro or in vivo $[9,10]$. Surface metrology is the measurement of small-scale characteristics from its intended shape. Surface topography consists of three basic components (form, waviness, and roughness). Roughness is what remains when the form and waviness components have been subtracted from the real contour of the surface. One advanced research regarding surface roughness is the use of implants in the dental field. This is normally characterized by some surface roughness parameters, and a textual characterization has been recommended by Mann et al. [11] .

Recently, [12] have quantified textual changes of enamel surface by citric acid and have observed the demineralization and remineralization processes using CLSM. They characterized enamel surface changes using "Sa", the overall roughness of a surface. It can be defined as the arithmetical average value of all absolute distances of the roughness profile from the center line within the measuring length. Ra (2D), Rq (2D), Rt (2D), Rc (2D), Sa (3D), Sq (3D), St (3D), and Sc (3D) are most commonly used parameters. However, these

Table 1: Description of test samples used for sampling procedure.

\begin{tabular}{ccc}
\hline Classification & Food item & Source \\
\hline Potatoes & Baked potato & Priginal sampling weight of the item \\
& Baked sweet potato & Sweet Potatoes, generic, raw \\
\hline Chips and cookies & Fried potato chip & Potato chips, Nongsim \\
& Baked potato chip & Yegam, Dongyang \\
& Butter cookie & Butter-ring, Haetae \\
\hline Bread & Bread, white & White Bread, Paris Baguette \\
& Sponge cake & Sponge Cake, Paris Baguette \\
Cereal & Doughnuts & Doughnuts, Paris Baguette \\
\hline Fruit & Cornflakes & Cornflakes, Nongsim-Kellogg's \\
\hline
\end{tabular}

Note: Preparation method for sampling is provided in the AOAC method by each individual food.

\section{Strain Preparation and Establishing the Pertinent Incu- bation Time}

Streptococcus mutans (ATCC 25715) was supplied by Korea Research Institute Bioscience and Biotechnology (KRIBB). This procedure was followed by the by Lee et al. [16] .

\section{Artificial Saliva Preparation}

Artificial saliva was formulated in $150 \mathrm{ml}$ of $\mathrm{Na}-\mathrm{CMC}$ (sodiumcarboxylmethlycellulose, Sigma-Aldrich Chemical Co., St. Louis, MO, USA), $30 \mathrm{ml}$ of glycerin, and $12 \mathrm{ml}$ of distilled water (Seoul National University Hospital formula). Na-CMC solution was manufactured as follows: $11.0 \mathrm{~g}$ of CMC-sodium, $1.0 \mathrm{~g}$ of methyl parabenzoate, $60 \mathrm{ml}$ of glycerin, and $5 \mathrm{ml}$ of ethanol were dissolved with distilled water in $1 \mathrm{~L}$ volumetric flask and autoclaved at $121^{\circ} \mathrm{C}, 15 \mathrm{lb}$ for 15 minutes . conventional parameters have limitations as predictors in the dental roughness profile. In particular, Ra was found to be insignificant regarding function by Thomas [13]. Hasson and Hansson [14] have suggested alternative parameters, such as skewness and kurtosis, because traditional parameters cannot discriminate surfaces. Austin et al. [12] have Have also mentioned that it is important to use the best surface parameters to characterize the demineralization process using appropriate instrumentation and replicating materials. Therefore, the objective of this study was to characterize the degree of enamel demineralization based on both optimized areal surface profiling and topography analysis using CLSM in vitro.

\section{Materials and Methods \\ Food Sample Preparation}

A total of 11 test samples were selected in the Food Logistics Almanac (Korea Ministry of Agriculture, Food, and Rural Affairs., 2011).The classification, item, source, and original weight of the material for sampling are summarized in Table 1. A total of 11 test samples were classified into 5 types. A modified AOAC method was used for sample preparation [15]. The standard sugar concentrations were set to $0.5 \%, 2 \%, 5 \%$, and $10 \%$. Sucrose $(10 \%)$ was used as a control.
\end{abstract}

\footnotetext{
.
} 
radioactive polyethylene bottle. ${ }^{32} \mathrm{P}$ released from radioisotopelabeled PAHA discs was measured with a liquid scintillation counter (LS 5000 TA, Beckman Instruments Inc., Fullerton, CA, USA) 3 times repeatedly.

\section{CLSM Analysis}

After the centrifugation from the liquid scintillation counter procedure, the remaining radioisotope-labeled PAHA discs were analyzed with CLSM. This procedure was followed by Lee et al. [17].

\section{SEM Analysis}

SEM image analysis was performed using S-4700 (Hitachi Ltd. Tokyo, Japan) at $150 \mathrm{x}$ magnification. All assays were repeated 10 times to reduce the disparity in the released amount of ${ }_{32} \mathrm{P}$ radioisotope, including the $10 \%$ sucrose, as a control.

\section{Statistical Analysis}

Statistical analysis was performed using SPSS, Windows (Version 23.0, IBM, Chicago IL, USA), and IBM Watson Analytics. With a significance rate of $p<0.05$, the data was expressed as the mean standard deviation (SD). Every attribute in surface roughness was selected as a variable. The amount of ${ }^{32} \mathrm{P}$ released from PAHA was statistically analyzed using one-way analysis of variance (ANOVA) and the Pearson correlation coefficient (r).

\section{Results}

\section{Demineralization Quantification}

Demineralization quantification was analyzed to the degree of ${ }^{32} \mathrm{P}$ released from radioisotope-labeled PAHA discs (Table 2). For the control group, the standard sugar concentration was set as $0.5 \%, 2 \%, 5 \%$, or $10 \%$. Based on the unit of released radioisotope, average amounts at sucrose concentrations of $0.5 \%, 2 \%, 5 \%$, and $10 \%$ were $7,555 \mathrm{cpm}, 8,010 \mathrm{cpm}, 8,124 \mathrm{cpm}$, and 10,027 $\mathrm{cpm}$, respectively. Demineralized amounts of ${ }^{32} \mathrm{P}$ released from PAHA discs exposed to prepared food samples are summarized in Table 2. Sucrose concentration at $10 \%$ showed the minimum value of standard deviation. Therefore, it was used as a control for measuring the cariogenic potential. The fruit group showed the highest degree of demineralized ${ }^{32} \mathrm{P}$ at an average value of apple 20,002.3 cpm and banana 18,677.3 cpm. Cereal presented an average released radioisotope of $16,578.7 \mathrm{cpm}$, which was less than that of the fruit group. The average of released radioisotope for combined groups of bread, chips, and cookies was 15,093.3 cpm. Average values of released radioisotope for sponge cake, baked potato chips, doughnuts, and white bread were at 13,692.6 cpm. Potato chips $(14,296 \mathrm{cpm})$ presented distinctive significance regarding demineralization. This might be due to its processing method and was extended to baked potato. This study did not analyze raw potato because it was rarely eaten by people, while baked potatoes and sweet potatoes were investigated (1,067.4 cpm for baked sweet potatoes and $977 \mathrm{cpm}$ for baked potatoes). These results indicated that unprocessed organic foods were less susceptible to demineralization. With regard to the control group, $10 \%$ sucrose showed a value of $10,027.4 \mathrm{cpm}$. These results showed distinguishable classifications between food groups based on demineralization in vitro.

Table 2: Demineralized amounts of phosphorus radioisotope (32P) released from polyacrylamide (PAHA) discs exposed to prepared food samples.

\begin{tabular}{|c|c|c|c|}
\hline \multirow{2}{*}{ Classification } & \multirow{2}{*}{ Food sample } & \multicolumn{2}{|c|}{ Demineralized ${ }^{32} \mathrm{P}^{\mathrm{b}}$} \\
\hline & & Quantity (cpm $\left.{ }^{\mathrm{c}}\right)$ & Ratio* \\
\hline Control & $10 \%$ sucrose solution & $10,027.4 \pm^{\mathrm{a}} 927.5$ & 1.00 \\
\hline \multirow{3}{*}{ Potatoes } & Baked potatoes & $978.1 \pm 102.0$ & 0.10 \\
\hline & Baked sweet potatoes & $1,068.1 \pm 109.6$ & 0.11 \\
\hline & Classification Total & $1,023.1 \pm 105.8$ & 0.11 \\
\hline \multirow{4}{*}{ Chips \& Cookies } & Potato chips & $14,296.0 \pm 2,098.0$ & 1.43 \\
\hline & Baked potato chips & $14,736.8 \pm 2,785.1$ & 1.47 \\
\hline & Butter cookies & $15,094.1 \pm 2,032.0$ & 1.50 \\
\hline & Classification Total & $14,709.0 \pm 2,035.0$ & 1.47 \\
\hline \multirow{4}{*}{ Bread } & White bread & $12,362.0 \pm 1,014.2$ & 1.23 \\
\hline & Sponge cake & $14,792.4 \pm 1,308.8$ & 1.48 \\
\hline & Doughnuts & $13,923.5 \pm 1,247.2$ & 1.39 \\
\hline & Classification Total & $13,692.6 \pm 1,190.1$ & 1.37 \\
\hline \multirow[t]{2}{*}{ Cereal } & Cornflakes & $16,579.0 \pm 2,229.1$ & 1.65 \\
\hline & Apples & $20,003.1 \pm 1,857.2$ & 2.00 \\
\hline \multirow[t]{2}{*}{ Fruit } & Bananas & $18,677.3 \pm 1,701.5$ & 1.86 \\
\hline & Classification Total & $19,340.2 \pm 1,779.4$ & 1.93 \\
\hline
\end{tabular}


a standard deviation.

${ }^{\mathrm{b}}$ Demineralized ${ }_{32} \mathrm{P}$ ratio $=$ demineralized ${ }^{32} \mathrm{P}$ amount for the test food $/$ demineralized ${ }^{32} \mathrm{P}$ amount for the standard $(10 \%$ sucrose) .

${ }^{\mathrm{c}} \mathrm{cpm}$ : counts per minute (unit of ${ }^{32} \mathrm{P}$ as measured by liquid scintillation counting).

*: $\mathrm{p}<0.05$ (DM-ANOVA of quantity of demineralized ${ }^{32} \mathrm{P}$ ).

p-value $\left(0.032^{*}\right)$ for all classification totals compared to control.

\section{Surface Roughness Quantification}

Variations in surface roughness parameters are summarized in Table 3. Amplitude parameters, including Sp (maximum peak height), Sv in valley depth, and Sz (maximum height of the surface) were evaluated from the absolute highest and lowest points within the surface. Butter cookies showed the greatest increase in variation $(\Delta=10.08 \mu \mathrm{m})$ of $\mathrm{Sp}$, while baked potatoes presented the most negative difference $(\Delta=-1.07 \mu \mathrm{m})$. Cornflakes showed the greatest decrease in maximum variation $(\Delta=-1.07 \mu \mathrm{m})$ in valley depth. However, butter cookies presented the greatest increase in value $(\Delta=6.37 \mu \mathrm{m})$. With regard to the maximum height of the surface, baked potatoes showed the most negative variation $(\Delta=-1.68$ $\mu \mathrm{m})$, while butter cookie remained as the candidate for increased variation $(\Delta=5.64 \mu \mathrm{m})$. Butter cookies also showed the highest variation $(\Delta=1.22 \mu \mathrm{m})$ in Sa (arithmetic mean height of the surface),

Table 3: Variation of surface roughness parameters. while potatoes presented modest difference at $0.03 \mu \mathrm{m}$. Sq (root square mean height of the surface) is the SD of surface roughness. Likewise, butter cookies presented the most positive variation $(\Delta=$ $1.70 \mu \mathrm{m})$. However, cornflakes were found to have the most depleted variant $(\Delta=-0.05 \mu \mathrm{m})$. All test samples showed decreased values in a variation of Sc (mean height of the surface of profile elements in a sampling). Baked sweet potatoes had the highest variation among samples at $-0.28 \mu \mathrm{m}$. Doughnuts showed sole positive variance at the minute degree of $\Delta=0.076 \mu \mathrm{m}$. The range of difference between food subjects was minuscule $(-0.025$ to- 0.276$)$ compared to other parameters. St and Smax parameters representing the total height of the surface and maximum roughness depth, respectively. Butter cookies and baked potato chips had the most positive deviation at $\Delta$ $=35.11 \mu \mathrm{m}$ in St and $\Delta=10.28 \mu \mathrm{m}$ in Smax, respectively. However, cornflakes showed negative variation at $\Delta=-2.21 \mu \mathrm{m}$ in St and $\Delta=$ $-4.34 \mu \mathrm{m}$ in Smax.

\begin{tabular}{|c|c|c|c|c|c|c|c|c|c|c|}
\hline Food items & $\mathrm{Sz}^{*}$ & $\mathrm{Sp}^{*}$ & $\mathbf{S v}^{*}$ & $\mathrm{Sa}^{*}$ & $\mathbf{S q}^{*}$ & $\mathrm{St}^{*}$ & Smax* & $\mathrm{Sc}^{*}$ & Ssk* & Sku* \\
\hline Baked potatoes & -1.68 & -1.07 & -0.01 & 0.03 & -0.05 & -1.07 & -1.78 & -0.03 & -0.65 & -1.19 \\
\hline Baked sweet potatoes & -0.64 & -0.80 & 0.31 & 0.18 & 0.19 & -0.49 & -1.90 & -0.28 & -0.20 & -0.45 \\
\hline Potato chips & 2.78 & 6.03 & 3.90 & 0.45 & 0.57 & 9.93 & 5.32 & 0.00 & -0.13 & 0.13 \\
\hline Baked potato chips & 4.01 & 2.48 & 7.44 & 0.30 & 0.46 & 9.92 & 10.28 & -0.03 & 0.61 & 5.98 \\
\hline Butter cookies & 10.08 & 12.41 & 22.70 & 1.54 & 1.89 & 35.11 & 5.32 & -0.08 & -0.07 & 1.59 \\
\hline White bread & 3.29 & 1.06 & 5.32 & 0.24 & 0.33 & 6.39 & 6.39 & -0.03 & 0.62 & 1.64 \\
\hline Sponge cake & 4.13 & -0.73 & 6.37 & 0.22 & 0.45 & 5.64 & 6.71 & -0.09 & 0.99 & 2.99 \\
\hline Doughnuts & 1.20 & -1.06 & 6.41 & 0.18 & 0.26 & 5.35 & 6.41 & 0.08 & 0.25 & 4.63 \\
\hline Cornflakes & 3.70 & 7.44 & -9.65 & 0.60 & 0.67 & -2.21 & -4.34 & -0.19 & -3.21 & -30.94 \\
\hline Apples & 5.62 & 3.55 & 5.67 & 1.22 & 1.70 & 9.22 & 9.58 & -0.05 & -0.06 & -3.83 \\
\hline Bananas & 5.64 & 4.23 & 4.91 & 1.08 & 1.25 & 9.14 & 7.73 & -0.24 & -1.04 & -4.48 \\
\hline
\end{tabular}

Legend: Sz, maximum height of the surface; Sp, maximum peak height; Sv, maximum valley depth; Sa, arithmetic mean height; Sq, root mean square height; St, total height; Smax, maximum roughness depth; Sc, mean height of the roughness profile elements; Ssk, skewness; Sku, kurtosis.

\section{Demineralized Surface Characterization}

Figure 1 highlighted a representative image of the demineralization effect of food groups and control through observation of subsurface lesions in PAHA discs using CLSM and SEM. The 10\% sucrose-induced PAHA disc had increased surface roughness with the presence of deep crevices. Surface lesions of potatoes had far fewer crevices. These were less irregular than baseline lesions. Beyond microscopic investigation, this study provided the degree of surface roughness data of both 3D and 2D parameters. Further demineralization quantification and cariogenic potential analysis should be based on 3D results because 3D surface topography represents both smooth and rough surfaces in optical profiling. However, 2D results were limited to singular surface profiling. The demineralized quantity may reflect sufficient evidence regarding the cariogenic potential of foods. 


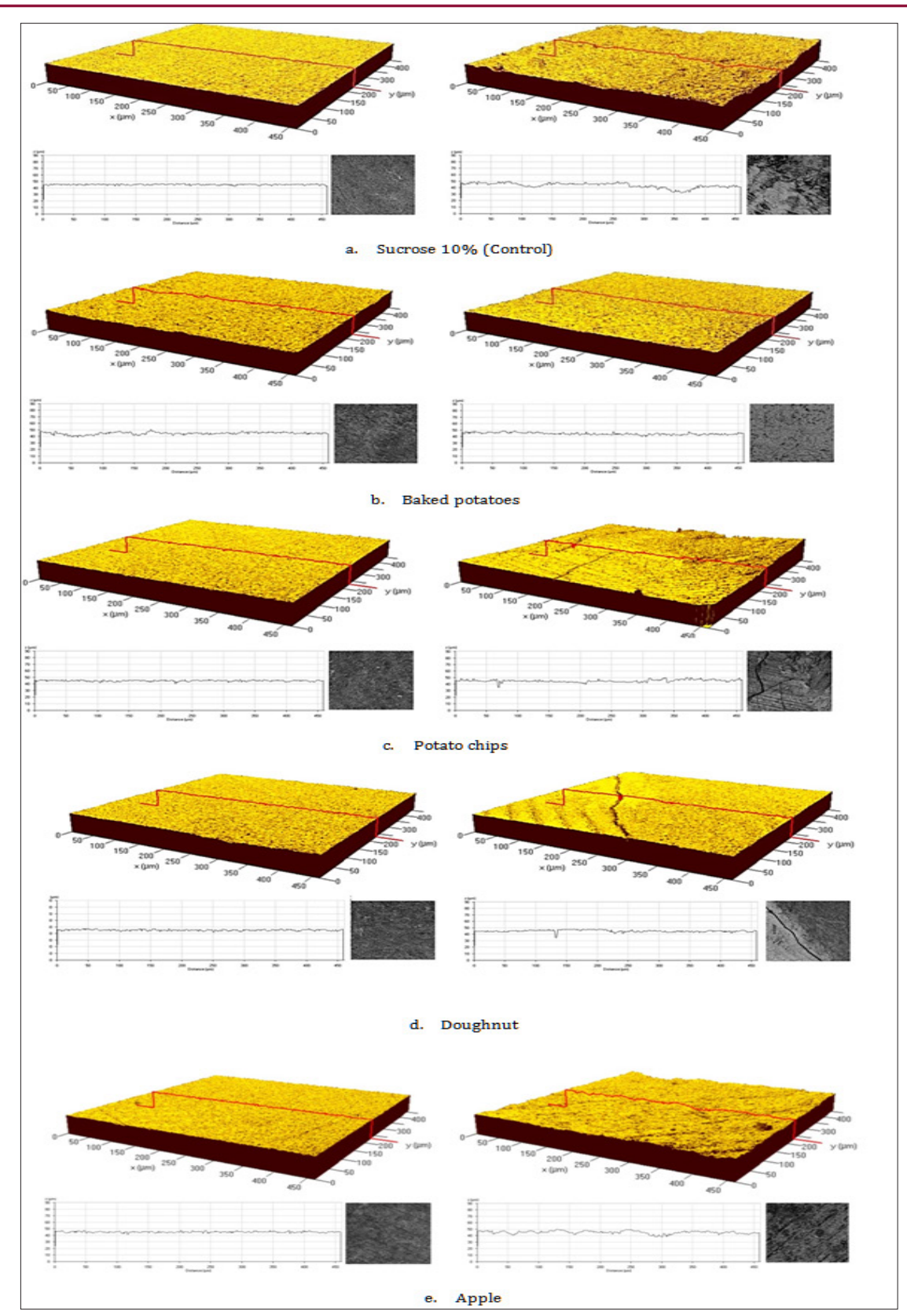

Figure 1: A representative image of the demineralization effect of snacks and control (10\% sucrose) through observation of subsurface lesions in polyacrylamide hydroxyapatite disc using confocal laser scanning microscopy and scanning electron microscopy. Left: Before; Right: After.

This study extended this viewpoint. Results for correlation between surface roughness parameter variation and demineralized ${ }^{32} \mathrm{P}$ as a dependent variable are shown in Table 4 . This result indicated that $\mathrm{Sz}$ showed a highly significant difference at $\mathrm{p}<$ 0.01 from demineralized ${ }^{32} \mathrm{P}$. Attributes related to mean height of the surface, such as Sa and Sq, presented significance at $\mathrm{p}<0.05$,

Table 4: Correlation of surface roughness parameters (variation) and demineralized ${ }^{32} \mathrm{P}$ (liquid scintillation count) as dependent variable.

\begin{tabular}{|c|c|c|c|c|c|c|c|c|}
\hline & $\mathbf{S z}^{* *}$ & Sp & Sv & Sa* & $\mathrm{Sq}^{*}$ & St & Smax & Sc \\
\hline Pearson correlation coefficient (r) & 0.76 & 0.50 & 0.23 & 0.61 & 0.66 & 0.39 & 0.41 & 0.13 \\
\hline Significance & 0.01 & 0.12 & 0.49 & 0.05 & 0.03 & 0.24 & 0.21 & 0.69 \\
\hline
\end{tabular}

put ${ }^{*} \mathrm{p}<0.05 ;{ }^{* *} \mathrm{p}<0.01$ reflecting their general usage in diverse fields. Among these three attributes, a correlation was found between the degree and vector of variation. An abnormality of texture roughness was found in cookies. This deviated the comparability with demineralization, although general affinity was found for other groups. 


\section{Discussion}

CLSM was presented in 3D and 2D layout. Because 2D parameters were deprived in a real surface profiling, 3D parameters were chosen for further investigation of smoothness, optical profiling, and characterization of the surface. A scale-dependent relative area analysis was used for micro wear measurement in the anthropological field to characterize dentin surface events during the initiation of acid production and affection $[9,11,18]$. Austin et al. and Scott et al. $[12,18]$ have demonstrated the effectiveness of areal surface texture analysis for characterizing enamel remineralization with CLSM. Because of their non-intrusiveness, CLSM has been highly utilized in recent advanced research studies to illustrate extrinsic factors in a simplistic manner $[12,19,20]$.

Despite the delicacy and difficulty in preparation and examination, this research provided SEM footage for comparative macroscopic analysis. As mentioned above, results of food sampleinduced PAHA [17] were analyzed with CLSM and described with 10 different parameters supported by a 3D image analysis system. Each attribute provided detailed evidence for demineralization quantification [Sa (common arithmetic mean height), Sq (root mean square height of the surface), Sp (maximum peak height), Sv (maximum valley depth), Sz (the maximum height of the surface)]. While fields and subjects, including biomaterials and dental implants, are regularly studied with diverse surface roughness parameters, full-scope optimization was not fully studied for oral biology studies [21,22].

Studies on the cariogenic potential of food have also remotely exploited such application. Ra \& Sa (arithmetic mean height of the surface roughness) and statistically stable $\mathrm{Rq}$ and Sq (root mean square height) are predominant due to their conventional usage and easy applicability. However, the Sa and Sq are overall average measures for texture finishing. Although singular attributes are generally used, these parameters are insensitive in discerning peaks, valleys, and spatial structures [23,24]. Moreover, there are a variety of general topographies. This study revealed that parameters other than those indicating the mean surface height showed distinctive aspects for 11 test samples. Cookies also showed the highest variation in Sa. However, potatoes presented modest difference at $0 \mu \mathrm{m}$. Sq is the standard deviation of surface roughness. Thus, Sq was more significant, with more physical grounding than Sa. Although these two parameters are closely correlated, Sc expresses the mean height of the surface of profile elements in a sampling length. Peaks and valleys are identified with minimum heights and lengths. All test samples showed decreased value in the variation of Sc. Statistical results proved that the degree of demineralization presented significant difference among the maximum values of $\mathrm{Sz}, \mathrm{Sa}$, and Sq. Both maximum peak and valley depth may indicate difference. However, Sa lacked sensitivity of morphology. This could be considered a drawback due to characteristics of PAHA discs, such as fragility amid preparation $[17,25]$. With a similar value of released radioisotope in PAHA compared to sponge cake, potato chips presented dramatic positive variation in kurtosis. Kurtosis is a measurement of sharpness. It is useful for indicating the presence of either peak or valley defects (spikiness). Within the surface, it is the ratio of the mean of the biquadratic mean of height values and the fourth power of Sq. Different from skewness (Ssk), it detects whether sample spikes are distributed consistently and the degree of spikiness. In other words, the presence of inordinate peaks and valleys are presented as Sku $>3$, while Sku $<3$ refers to bumpy surface compared to beforehand.

Sponge cake and bread tended to have more peaks in comparison with apples. Skewness is the ratio of the mean of heights cubed and the cube of Sq within the surface. In other words, it expresses the symmetry of peaks and valleys based on the average central line. Values higher than 0 indicate the prevalence of peaks, while values lower than 0 imply valley topography. Ssk at 0.00 infers normal distribution (i.e., bell curve). Both bread and cookie groups showed a unified tendency toward a predominance of peaks. However, apples, sweet potatoes, potatoes, bananas, and cornflakes presented valley predominance. Compared to cornflakes, other food samples presented negative variance with fluctuating values of -30.94. Parameters such as skewness (Ssk) and kurtosis (Sku) presented predominance of peaks and valleys with the presence of inordinate peaks and valleys correspondingly.

\section{Conclusion}

This study confirmed the feasibility of quantified demineralization using both optimized profiling and surface topography in 11 foods. The $10 \%$ sucrose-induced PAHA discs had increased surface roughness with the presence of deep crevices. The skewness and kurtosis, distinct demineralization was observed for sponge cake $(p<0.05)$. The comparison of multiple parameters of CLSM provided more diverse quantities and degrees of demineralization. A fair amount of research related to demineralization has used $\mathrm{Sa}$ or $\mathrm{Sq}$ $(p<0.05)$. Although statistical evaluation may support the correlation between each parameter and demineralization degree (quantity) released from PAHA discs, further characterization by either skewness or kurtosis or both could illustrate such correlation. Still, the surface roughness is the most general amplitude parameter. Its attributes include functional parameters. This study suggests that surface morphology should be based on multiple parameters to understand characteristics and advantage points more clearly.

\section{References}

1. Curzon ME, Hefferren JJ (2001) Modern Methods for Assessing the Cariogenic and Erosive Potential of Foods. British Dental Journal 191(1): 41-46.

2. Featherstone JD, Zero DT (1992) An in situ Model for Simultaneous Assessment of Inhibition of Demineralization and Enhancement of Remineralization. Journal Dental Research 71(Spec): 804-810.

3. Fejerskov O, Nyvad B, Larsen MJ (1994) Human Experimental Caries Models: Intra-Oral Environmental Variability. Advances in Dental Research 8(2): 134-143.

4. Lingström P, Imfeld T, Birkhed D (1993) Comparison of Three Different Methods for Measurement of Plaque-Ph in Humans after Consumption of Soft Bread and Potato Chips. Journal Dental Research 72(5): 865-870.

5. Navia JM, Lopez H, Bradley EL (1981) Biological Rat Assay for Total Fluoride Availability in Foods. Journal of Nutrition 111(4): 755-761. 
6. Meurman JH, Frank RM (1991) Scanning Electron Microscopic Study of the Effect of Salivary Pellicle on Enamel Erosion. Caries Research 25(1): 1-6.

7. Mistry M, Grenby TH (1993) Erosion by Soft Drinks of Rat Molar Teeth Assessed by Digital Image Analysis. Caries Research 27(1): 21-25.

8. Edgar WM (1985) Prediction of the Cariogenicity of Various Foods. International Dental Journal 35(3): 190-194.

9. Clarkson BH, Wefel JS, Feagin FF (1986) Fluoride Distribution in Enamel after in Vitro Caries-Like Lesion Formation. Journal Dental Research 65(7): 963-966.

10. Schlueter N, Hara A, Shellis RP, Ganss C (2011) Methods for the Measurement and Characterization of Erosion in Enamel and Dentine. Caries Research 45 (Supplement 1): 13-23.

11. Mann C, Ranjitkar S, Lekkas D, Hall C, Kaidonis JA, et al. (2014) ThreeDimensional Profilometric Assessment of Early Enamel Erosion Simulating Gastric Regurgitation. Journal of Dentistry 42(11): 14111421.

12. Austin RS, Ungar PS, Bergstrom TS, Brown CA, Grine FE, et al. (2016) Confocal Laser Scanning Microscopy and Area-Scale Analysis Used to Quantify Enamel Surface Textural Changes from Citric Acid Demineralization and Salivary Remineralization in vitro. Dental Materials 32(2): 278-284.

13. Thomas TR (1981) Characterization of surface roughness. Precision Engineering 3(2): 97-104.

14. Hansson KN, Hansson S (2011) Skewness and Kurtosis: Important Parameters in the Characterization of Dental Implant Surface Roughness - a Computer Simulation. ISRN Materials Science 2011(2011): 6.

15. AOAC (1995) Official Method of Analysis of AOAC International, $\left(16^{\text {th }}\right.$ edn.), Association of Official Analytical Chemists Inc., Arlington, VA USA.
16. Lee EJ, Yun HK, Hwang Ik, Bae KH, Jin BH (2012) Measurement of the cariogenicity of snacks using a radioisotope PAHA disc. Archives of Oral Biology 57(6): 769-774.

17. Lee EJ, Hwang Ik, Paik DI, Jin BH (2015) The Cariogenicity of Hydrolyzed Starch Foods by Measuring the Polyacrylamide Hydroxyapatite (PAHA) Disc. Archives of Oral Biology 60(11): 1632-1638.

18. Scott RS, Ungar PS, Bergstrom TS, Brown CA, Grine FE, et al. (2005) Dental Microwear Texture Analysis Shows within-Species Diet Variability in Fossil Hominins. Nature 436: 693-695.

19. Lechner BD, Röper S, Messerschmidt J, Blume A, Magerle R (2015) Monitoring Demineralization and Subsequent Remineralization of Human Teeth at the Dentin-Enamel Junction with Atomic Force Microscopy. ACS Applied Materials \& Interfaces 7(34): 18937-18943.

20. Quartarone E, Mustarelli P, Poggio C, Lombardini M (2008) Surface Kinetic Roughening Caused by Dental Erosion: An Atomic Force Microscopy Study. Journal of Applied Physics 103: 104702.

21. Al Omari WM, Mitchell CA, Cunningham JL (2001) Surface Roughness and Wettability of Enamel and Dentine Surfaces Prepared with Different Dental Burs. Journal of Oral Rehabilitation 28(7): 645-650.

22. Elias CN, Oshida, Y, Lima JH, Muller CA (2008) Relationship between Surface Properties (Roughness, Wettability and Morphology) of Titanium and Dental Implant Removal Torque. Journal of the Mechanical Behavior of Biomedical Materials 1(3): 234-242.

23. Whitehouse DJ (1982) The Parameter Rash- Is There a Cure?. Wear 83(1): 75-78.

24. Whitehouse DJ (2002) Handbook of Surface and Nanometrology $\left(2^{\text {nd }}\right.$ edn), CRC Press, New York, USA.

25. Blunt L, Jiang X (2003) Advanced Techniques for Assessment Surface Topography: Development of a Basis for 3d Surface Texture Standards "Surfstand", Kogan Page Science, London, UK.
(C) This work is licensed under Creative

Submission Link: http://biomedres.us/submit-manuscript.php

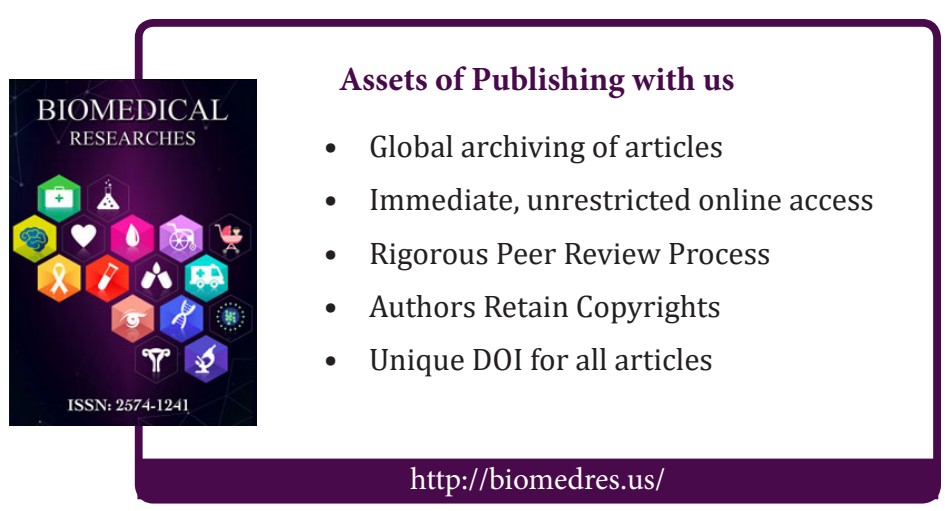

